\title{
Endomyocardial biopsies in patients with left ventricular hypertrophy and a common Chinese later-onset fabry mutation (IVS4 + 919G > A)
}

Ting-Rong Hsu ${ }^{1,2}$, Shih-Hsien Sung ${ }^{3}$, Fu-Pang Chang ${ }^{4}$, Chia-Feng Yang ${ }^{2}$, Hao-Chuan Liu² , Hsiang-Yu Lin ${ }^{5}$, Chun-Kai Huang 2,6, He-Jin Gao 2,6, Yu-Hsiu Huang 2,6, Hsuan-Chieh Liao7, Pi-Chang Lee², An-Hang Yang ${ }^{4}$, Chuan-Chi Chiang ${ }^{7}$, Ching-Yuang Lin ${ }^{8}$, Wen-Chung $\mathrm{Yu}^{3^{*}}$ and Dau-Ming Niu ${ }^{1,2,6^{*}}$

\begin{abstract}
Background: In Taiwan, DNA-based newborn screening showed a surprisingly high incidence of a cardiac Fabry mutation (IVS4 + 919G > A). The prevalence of this mutation is too high to be believed that it is a real pathogenic mutation. The purpose of this study is to identify the cardiac pathologic characteristics in patients with left ventricular hypertrophy and this mutation

Methods and results: Endomyocardial biopsies were obtained in 22 patients (Median age: 61, males: 17; females: 5) with left ventricular hypertrophy and the IVS4 + 919G > A mutation; five patients had not received enzyme replacement therapy (ERT) before biopsy, while the other 17 patients had received ERT from 8 months to 51 months. Except for three patients who had received ERT for more than 3 years, all other patients showed significant pathological change and globotriaosylceramide (Gb3) accumulation in their cardiomyocytes. In contrast to classical Fabry patients, no Gb3 accumulation was found in the capillary endothelial cells of any of our patients. Fourteen patients (63.6\%) were found to have myofibrillolysis.

Conclusions: All of the untreated and most of the treated IVS4 + 919G > A patients showed typical pathological changes of Fabry disease in their cardiomyocytes. No endothelial accumulation of Gb3 was found, which is similar to the findings of several previous reports regarding later-onset Fabry disease. This result highly suggests that the IVS4 + 919G > A is a real pathogenic later-onset Fabry mutation.
\end{abstract}

Keywords: Endomyocardial biopsy, Enzyme replacement therapy, Fabry disease, IVS4 + 919G > A, Left ventricular hypertrophy

\section{Background}

Fabry disease (MIM 301500) is an X-linked lysosomal storage disorder resulting from deficient alpha-galactosidase $\mathrm{A}$ $(\alpha-G a l$ A) activity. The estimated incidence of classic Fabry disease is 1 in 40,000-60,000 males in the general population [1]. The deficient $\alpha$-Gal A activity results in progressive accumulation of glycosphingolipid, predominantly globotriaosylceramide (Gb3), in the walls of small blood vessels, nerves, dorsal root ganglia, renal glomerular and tubular

\footnotetext{
* Correspondence: wcyu@vghtpe.gov.tw; dmniu1111@yahoo.com.tw ${ }^{3}$ Division of Cardiology, Department of Medicine, Taipei Veterans General Hospital and National Yang-Ming University, School of Medicine, No. 201, Section 2, Shih-Pai Road, 112 Taipei, Taiwan

${ }^{1}$ Institute of Clinical Medicine, National Yang-Ming University, Taipei, Taiwan Full list of author information is available at the end of the article
}

epithelial cells, and cardiomyocytes. Clinical features in classically affected patients include acroparesthesia, angiokeratoma, and hypohidrosis in early childhood or adolescence and progress to renal insufficiency, cardiomyopathy, and cerebrovascular disease in adulthood [2-6]. Recently, several different later-onset phenotypes of Fabry disease have been identified, which have drawn the attention of more physicians [7-9]. Patients with later-onset Fabry disease have higher residual enzyme activities than those of the classical type. They lack the classic symptoms of Fabry disease and present relatively fewer or isolated symptoms such as hypertrophic cardiomyopathy, renal failure, or cryptogenic stroke at later stages in life [10-14]. The cardiac later-onset phenotype usually presents only with 
cardiac manifestations, such as hypertrophic cardiomyopathy, mitral insufficiency and/or arrhythmias in the fifth to eighth decade $[12,15,16]$.

In Taiwan, our team first revealed a surprisingly high incidence (approximately 1 in 1600 males) of a later-onset GLA mutation, IVS4 + 919G > A (GenBank accession nos. CS020811), in our population [17] and also identified this mutation in a number of Taiwan Chinese adult patients with idiopathic hypertrophic cardiomyopathy [18]. Thereafter, another newborn screening center of Taiwan also revealed a very similar incidence ( 1 in 1,460 males) of this mutation in their study [19]. More recently, DNA-based newborn screening for this mutation revealed a higher incidence $(1 / 875$ in males and 1/399 in females) than our previous enzyme-based Fabry newborn screening in Taiwan [20]. Because of the high prevalence of this IVS4 + 919G > A mutation in our population [21,22], we analyzed the disease onset rate according to the ages of the male and female adults with this mutation. 86 male and 248 female adults who were older than 30 years were enrolled in this analysis. We found the disease onset rate for both male and female patients increased gradually with increasing age (Figure 1). Around $77 \%$ of male adults and $35 \%$ of female adults, who were older than 40 years old, had already developed hypertrophic cardiomyopathy. In particular, $94 \%(32 / 34)$ of the males who were older than 60 years old and $100 \%(6 / 6)$ who were older than 70 years old showed significant hypertrophic cardiomyopathy (Figure 1). We believe this result strongly suggests that this IVS4 + 919G $>$ A is a real pathogenic mutation. Although, the IVS4 + 919G > A mutation was found in such a high frequency, the pathologic changes in later-onset Fabry disease with this specific mutation is still poorly known. It is important to look into the pathologic characteristics and changes of cardiomyopathy in these patients [23].

Due to the high prevalence of IVS4 + 919G > A mutation in our population, a new treatment guideline has been set up by the Bureau of National Health Insurance in Taiwan. All Fabry patients applying for enzyme replacement therapy (ERT) funding from the national health insurance have to receive an endomyocardial biopsy examination to demonstrate that Fabry disease is the primary cause of their hypertrophic cardiomyopathy. This guideline gives us the opportunity to observe a large series of cardiac pathologic changes in these patients with left ventricular hypertrophy and the IVS4 + 919G > A mutation.

\section{Patients and methods}

\section{Patient population}

A total of 22 Fabry patients (17 males and 5 females, median age: 61 years old) with left ventricular hypertrophy carrying the IVS4 + 919G > A mutation were enrolled in this study. The demographic data, laboratory results, image results and pathologic findings of the endomyocardial biopsies were collected retrospectively from December 2012 to April 2013.

Five patients (nos.1-5) did not receive ERT before endomyocardial biopsy. The others had received agalsidase alfa (Replagal ${ }^{\circ}$, Shire) $0.2 \mathrm{mg} / \mathrm{kg}$ every other week as the enzyme replacement therapy for at least 8 months (range 840 months). Two patients (nos. 16 and 17) had initially received agalsidase beta (Fabrazyme ${ }^{\circ}$, Genzyme) $1 \mathrm{mg} / \mathrm{kg}$ every other week and then changed to Replagal ${ }^{\circ} 0.2 \mathrm{mg} / \mathrm{kg}$ in 2009 owing to the global shortage of Fabrazyme.

The study complies with the Declaration of Helsinki and is approved by the medical ethics committee of Taipei

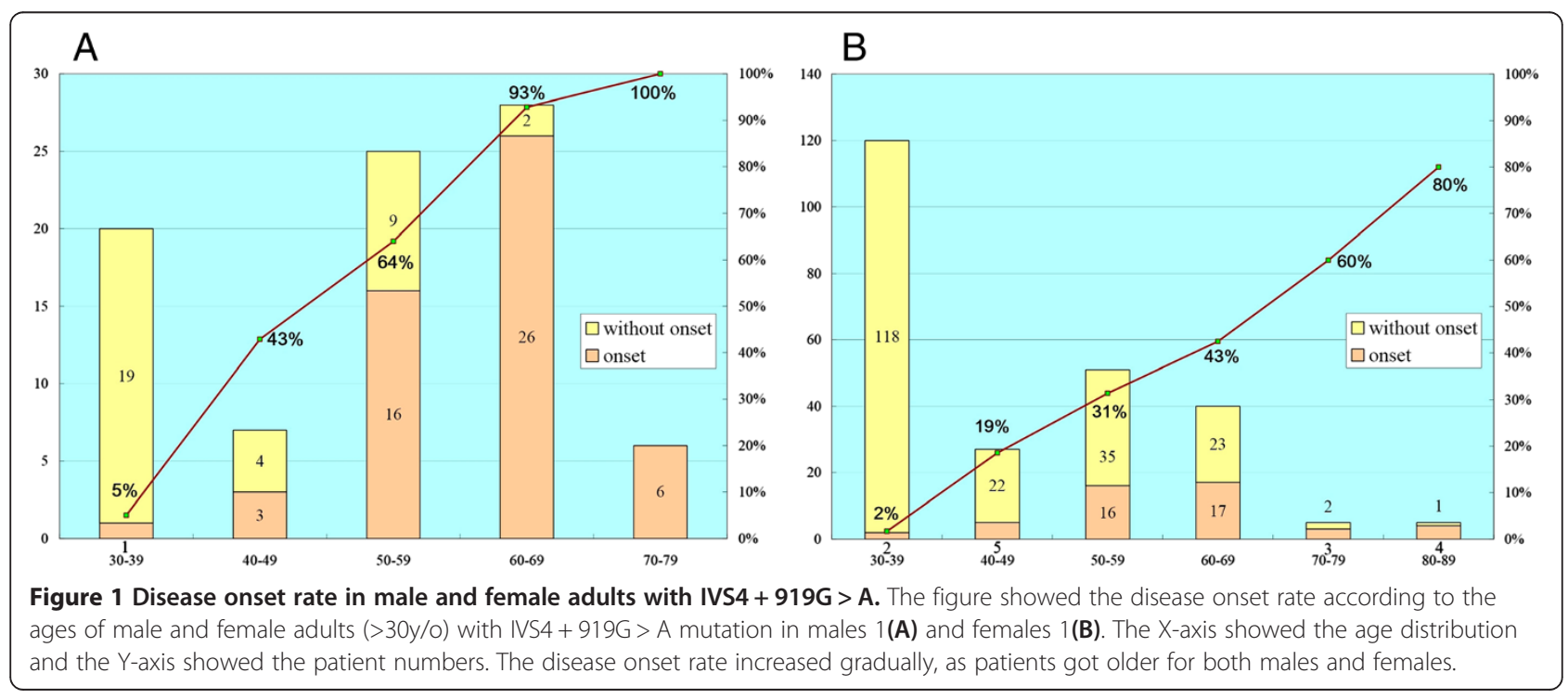


Veteran General Hospital. Written informed consents were obtained.

\section{GLA gene mutation}

Blood samples were obtained from these patients in blood collecting tubes containing ethylene diamine tetraacetic acid, and samples were stored at $4^{\circ} \mathrm{C}$. DNA was isolated from whole blood using the GFX genomic Blood DNA Purification Kit (Amersham Biosciences, UK) following the manufacturer's instructions. The primer sets were used to amplify the sequences of seven GLA exons and the region including IVS4 + 919G > A $[24,25]$. The polymerase chain reaction products were analyzed by $1.5 \%$ agarose I (Amresco) gel electrophoresis and then eluted in the polymerase chain reaction Advanced PCR Clean Up System (Viogene, USA.). Direct sequencing of the $\alpha$ Gal A gene was processed using the BigDye Terminator v3.1 Cycle Sequencing Kit (Applied Biosystems) and ABI Prism 3730 Sequencer [26].

\section{Plasma a-Gal A activity and Plasma LysoGb3}

Plasma $\alpha-G a l$ A activity was determined using the substrate 4-methylumbelliferyl $\alpha$-D-galactopyranoside $(5 \mathrm{mmol} / \mathrm{L}$ ) freshly prepared in $117 \mathrm{mmol} / \mathrm{L} \mathrm{N}$-acetyl-D-galactosamine/ $50 \mathrm{mmol} / \mathrm{L}$ citric-phosphate buffer, $\mathrm{pH}$ 4.6, before every assay. In brief, $50 \mu \mathrm{L}$ of plasma was mixed with $300 \mu \mathrm{L}$ of the substrate solution, incubated at $37^{\circ} \mathrm{C}$ for 2 hours, and $0.2 \mathrm{~N}$ glycine- $\mathrm{NaOH}$ was added to stop the reaction. Fluorescence intensity was measured with the excitation and emission wavelengths of 365 and $450 \mu \mathrm{m}$, respectively $[27,28]$. Plasma LysoGb3 was detected by tandem mass spectrometry that was performed in positive ion mode $(\mathrm{ES}+)$ on a triple quadruple mass spectrometer (Quattro Ultima, Waters, Milford, MA) with NeoLynx software version 4.1. A multiple reaction monitoring (MRM) mode was used for the measurement of lysoGb3. The analyzing methods were modified from the protocol provided by Shire [29].

\section{Echocardiography}

Echocardiography was performed routinely for the Fabry patients to check the hypertrophic cardiomyopathy. Left ventricular mass was calculated according to the Devereux cube formula and indexed to height ${ }^{2.7}$ (LVMI). Left ventricular hypertrophy by echocardiography was defined by a LVMI of $>47 \mathrm{~g} / \mathrm{m}^{2.7}$ in women or $>50 \mathrm{~g} / \mathrm{m}^{2.7}$ in men [30-32]. To avoid variability of LMVI measurements, only the results performed by two cardiologists specializing in echocardiography and measured with the same machine and protocol were used in this study.

\section{Cardiac magnetic resonance imaging}

Magnetic resonance imaging (MRI) of the heart was carried out as part of stand assessment on a $1.5 \mathrm{~T}$ scanner
(Excite II; GE medical systems, Milwaukee, WI). Late enhancement (LE) technique by gadolinium-contrast cardiac MRI examination was applied for the later-onset Fabry patients for assessment of fibrosis. The participants underwent a scan with spin echo double IR T1WI and T2WI pulse sequences and Fiesta pulse sequence, on axial, coronal, and variable scanning plans. Images were acquired 10 minutes after the intravenous injection of gadolinium. The myocardial delayed enhancement protocol included the pre-Gd axial FIESTA cine and grid tagging in $8 \mathrm{~mm}$ slice, and the post-Gd myocardial delayed enhancement of short axis/fourchamber views in $8-\mathrm{mm}$ slice and oblique axial cine of ascending aorta in $8-\mathrm{mm}$ slice to detect changes in tissue integrity in the left ventricle myocardium [33-35]. Severe myocardial fibrosis was defined by at least two affected left ventricular segments, which are followed by the American heart association guideline [36], and mild myocardial fibrosis was defined by one segment affected.

\section{Cardiac catheterization and endomyocardial biopsy}

Right heart catheterization was approached via the right internal jugular vein under digital X-ray guidance. A flexible endomyocardial bioptome was inserted into the right ventricle and 2-3 specimens were obtained from interventricular septum and submitted to histological examination.

\section{Histological studies}

Cardiac specimens selected for light microscope were fixed in $10 \%$ buffered formalin, embedded in paraffin. The sections were cut from the paraffin block and stained with haematoxylin and eosin $(\mathrm{H} \& \mathrm{E})$, and Masson's trichrome. Cardiac specimens selected for electron microscope were fixed in $2.5 \%$ glutaraldehyde in phosphate buffer, postfixed with $1 \%$ OsO4 in Sorenson's phosphate buffer, followed by dehydration through a graded series of ethanol washes, and embedded in Spurr's EPON. Semithin sections were cut from the block and stained with toluidine blue for adequate preview under a microscope. Ultrathin sections were prepared and examined under an electron microscope.

In the idealized model of a cardiomyocyte, as an elliptic cylinder, different diameters such as major axis (longest diameter) or minor axis (shortest diameter), etc. are measured, even in one transverse section of a cardiomyocyte. The current study revealed that, in the transverse section, the ratio between the major axis and minor axis of the cardiomyocytes is usually around 1.1 $(11 / 10)$ [37]. Therefore, the diameters of cardiomyocytes in transverse section can be approximately estimated. Because the minor axis of the transverse section of cardiomyocytes can be measured more easily and reliably 
than other diameters of the other axis, the diameter of the minor axis of the transverse section of cardiomyocytes was used to present the diameter of cardiomyocytes in several previous reports [38-41]. In accordance with these previous reports, we measured the minor axis at the nuclear level in transverse section to present the diameter of cardiomyocytes of our patients in this study. A total of 15 cells from 3 micrographs were measured for each sample. Control samples were retrospectively obtained from 8 anonymous patients who were provided by the department of pathology in our medical center. All the samples were obtained during the electrophysiology study for arrhythmia. None of these patients had any evidence of hypertrophic cardiomyopathy. All the histopathologic specimens were blindly reviewed by the same pathologist.

\section{Data analysis and statistics}

The data in some parts of the study were analyzed with descriptive statistics (mean, standard deviation). Due to the small number of patients and the uncontrolled nature of this study, no inferential statistics were used. Results are presented as actual measurements from individual patients. SPSS Version 20 (SPSS Inc, Chicago, Illinois, USA) was used for descriptive statistics.

\section{Results}

The demographic data and clinical manifestations of these patients are shown in Table 1. Interestingly, high prevalences of hypertension and hyperlipidemia were noted in these IVS4 + 919G > A patients with hypertrophic cardiomyopathy. Out of these 22 patients, 12 (54.5\%) were noted to have hypertension. All of these hypertensive patients received anti-hypertensive treatment and were kept normotensive $(<140 / 90 \mathrm{mmHg})$ for at least 6 months before commencement of ERT. Seven (31.8\%) patients were found to have hyperlipidemia and all of them were well controlled by medication for at least 6 months.

Twenty out of 22 patients received gadolinium-contrast enhancement cardiac MRI with LE technique and 6 patients $(30.0 \%)$ were found to have severe fibrosis which is defined as at least 2 segments that are positive in myocardium delayed enhancement scan.

All the endomyocardial biopsies were successfully performed without complication. For the pathologic change of the endomyocardial biopsies, all 22 patients (100\%) showed myocardial hypertrophic change with distorted nuclei, and increased cardiomyocyte size with $\mathrm{H} \& \mathrm{E}$ staining. Five $(100 \%)$ of the patients who did not receive ERT showed diffuse vacuolization in their myocardiocytes (Figure 2A) which is a common pathologic finding for Gb3 accumulation in cardiac biopsies of Fabry patients. In the other 17 patients who had received ERT, except for 3 patients (nos. 16, 17 and 22) who had received ERT for more than 3 years (Figure 3), all of them also showed diffuse Gb3 accumulation in cardiomyocytes. Regarding the toluidine blue staining, except those 3 patients (nos. 16,17 and 22), the other 19 patients were also noted to have a lot of dark blue substance accumulation in cardiomyocytes, which is consistent with Gb3 accumulation (Figure $2 \mathrm{C}$ and $\mathrm{D}$ ). In addition to Gb3 accumulation, patient no. 10 was found to have interstitial fibrosis among the cardiomyocytes with $\mathrm{H} \& \mathrm{E}$ staining (Figure 2B). Regarding the electron microscopic examination, except for those three patients, all other patients were found to have abundant lamellar myelin bodies in their cardiomyocytes (Figure 4). Furthermore, 14 patients (63.6\%) were found to have significant myofibrillolysis (Figure 4D). There was no Gb3 accumulation in the capillary endothelium of any of these patients (Figure 4E, and F), which is compatible with the histopathologic finding of later-onset Fabry disease.

The diameters of cardiomyocytes were measured in all 22 patients (Figure 5). The average diameter of the cardiomyocytes was $25.35 \pm 6.02 \mu \mathrm{m}$. The result of each individual is shown in Table 1. The average diameter of the control cardiomyocytes was $14.89 \pm 2.63 \mu \mathrm{m}$.

\section{Discussion}

Recently, the later-onset phenotypes of Fabry disease were found to be about 10 times more frequent in most populations $[1,20,42,43]$. Owing to the characteristics, the terminology "later-onset" Fabry disease might be more appropriate than "variant" Fabry disease. In fact, an increasing number of novel mutations have been found and believed to be later-onset mutations through high-risk patient screenings or newborn screenings. On the other hand, some mutations, which were previously thought to be later-onset mutations, have been proved to be non-pathogenic. The most noticeable example is the p,E66Q mutation (c.196G > C), a previously identified renal later onset type mutation, which has a very high prevalence rate in Japanese and Korean populations $(0.5-1 \%)$ and which has now been proved by pathologic studies to be merely a single nucleotide polymorphism, not a pathogenic mutation [44]. Therefore, it is insufficient to confirm a later onset mutation based on its enzyme activity and clinical manifestations such as hypertrophic cardiomyopathy or renal impairment.

Biomarkers, such as Gb3 or lyso-Gb3 were considered to be helpful to determine if the novel mutation is a true pathogenic mutation [27]. However, several reports indicated that Gb3, and even lysoGb3 might be normal in certain Fabry patients who had developed significant clinical manifestations with the pathogenic mutations $[45,46]$. Renal or cardiac biopsy can provide more information to identify if a novel mutation is a real pathogenic mutation. In the retrospective single-center direct observational study, 
Table 1 The demographic data and clinical manifestations of the Fabry disease patients with IVS4 + 919G > A mutation

\begin{tabular}{|c|c|c|c|c|c|c|c|c|c|c|c|c|c|c|c|}
\hline \multirow[t]{2}{*}{ Pt } & \multirow[t]{2}{*}{ Age } & \multirow[t]{2}{*}{ Sex } & \multirow[t]{2}{*}{ Comorbidity } & \multicolumn{5}{|c|}{ Histology ${ }^{a}$} & \multirow{2}{*}{$\begin{array}{c}a-G a l \\
A^{c}\end{array}$} & \multirow[t]{2}{*}{ LysoGb3 $^{d}$} & \multicolumn{2}{|c|}{ LVMI $^{\mathrm{e}}$} & \multirow[t]{2}{*}{$\mathrm{Cr} / \mathrm{eGEF}^{\mathrm{f}}$} & \multirow[t]{2}{*}{$\mathrm{LE}^{\mathrm{g}}$} & \multirow[t]{2}{*}{ ERT $^{\mathrm{i}} /$ Dose $^{\mathrm{j}}$} \\
\hline & & & & $\mathrm{HC}$ & MB & MFL & Other & Diameter & & & Initial & biopsy & & & \\
\hline 1 & 58 & M & Hyperlipidemia; Hypertension & + & + & + & & $34.7 \pm 4.4$ & 1.35 & 3.21 & 57.0 & 57.0 & $0.88 / 94.5$ & No & Not yet/0 \\
\hline 2 & 61 & M & Hyperlipidemia; Hepatitis B & + & + & + & & $21.8 \pm 3.3$ & 2.75 & 6.51 & 66.9 & 66.9 & $0.92 / 88.9$ & severe & Not yet/0 \\
\hline 3 & 75 & M & $\begin{array}{l}\text { Coronary artery disease; Arrhythmia; } \\
\text { Hepatitis C; Cholecystitis }\end{array}$ & + & + & + & & $31.5 \pm 4.0$ & 1.15 & 6.51 & 83.0 & 83.0 & $1.29 / 57.7$ & $N A^{h}$ & Not yet/0 \\
\hline 4 & 66 & M & $\begin{array}{l}\text { DM; Hypertension;ESRD s/p renal transplantation; } \\
\text { Cholecystitis; Hyperuricemia }\end{array}$ & + & + & + & & $30.8 \pm 5.6$ & 1.29 & 7.13 & 154.9 & 154.9 & $1.77 / 41.1$ & severe & Not yet/0 \\
\hline 5 & 64 & M & $\begin{array}{l}\text { Hypertension; Coronary artery disease; } \\
\text { Hyperlipidemia; Hyperuricemia }\end{array}$ & + & + & + & & $33.5 \pm 8.0$ & 1.32 & 7.47 & 128.1 & 128.1 & $0.97 / 82.8$ & No & Not yet/0 \\
\hline 6 & 58 & M & Hyperlipidemia & + & + & - & & $24.1 \pm 2.4$ & 2.17 & 9.60 & 54.5 & 38.5 & $1.01 / 80.6$ & No & $8 \mathrm{M} / 3.80$ \\
\hline 7 & 61 & M & Cubital tunnel syndrome & + & + & + & & $25.9 \pm 2.8$ & 1.54 & 7.53 & 52.2 & 52.2 & $0.85 / 97.4$ & severe & $10 \mathrm{M} / 4.11$ \\
\hline 8 & 56 & M & Hypertension; DM; Duodenal ulcer & + & + & - & & $25.6 \pm 7.0$ & 1.39 & 6.13 & 74.5 & 74.8 & $0.92 / 90.5$ & No & $10 \mathrm{M} / 4.35$ \\
\hline 9 & 66 & M & Hyperlipidemia; Sick sinus syndrome & + & + & + & Fatty $^{b}$ & $24.9 \pm 3.6$ & 1.15 & 9.77 & 136.4 & 113.9 & $1.4 / 53.9$ & No & $1 Y 5 M / 7.53$ \\
\hline 10 & 52 & M & Coronary artery disease; Fatty liver & + & + & + & Fibrosis & $21.1 \pm 5.0$ & 0.94 & 11.27 & 54.8 & 61.0 & $0.86 / 99.3$ & No & $1 Y 6 \mathrm{M} / 8.18$ \\
\hline 11 & 62 & M & BPH; Bladder cancer; Osteoarthritis & + & + & - & & $21.6 \pm 4.0$ & 0.79 & 5.06 & 50.7 & 52.8 & $0.95 / 85.4$ & mild & $1 \mathrm{Y} 11 \mathrm{M} / 9.76$ \\
\hline 12 & 63 & M & Hyperlipidemia; Arrhythmia s/p pacemaker & + & + & - & & $23.2 \pm 3.4$ & 1.02 & 13.51 & 51.2 & 50.9 & $1.16 / 67.6$ & $N A^{h}$ & $2 Y 9 M / 14.90$ \\
\hline 13 & 58 & M & Hypertension; Hyperlipidemia & + & + & + & & $26.2 \pm 5.6$ & 1.32 & 5.44 & 50.5 & 41.0 & $1.15 / 69.4$ & mild & $2 \mathrm{Y} 10 \mathrm{M} / 15.61$ \\
\hline 14 & 58 & M & Hypertension & + & + & + & & $24.0 \pm 5.4$ & 1.30 & 8.22 & 66.5 & 83.7 & 1.1470 .1 & No & $2 \mathrm{Y} 11 \mathrm{M} / 16.33$ \\
\hline 15 & 66 & M & Hypertension, Hyperuricemia & + & + & + & & $24.2 \pm 4.1$ & 1.47 & 14.54 & 88.2 & 81.4 & $0.81 / 101.3$ & severe & $2 Y 11 M / 16.33$ \\
\hline 16 & 47 & M & Hypertension; Arrhythmia & + & Scanty & - & & $23.1 \pm 4.1$ & 0.65 & 6.16 & 92.1 & 74.9 & $0.8 / 110.1$ & severe & $4 \mathrm{Y} 3 \mathrm{M} / 44.84$ \\
\hline 17 & 58 & M & Hypertension; DM; BPH & + & No & - & & $23.6 \pm 3.8$ & 0.88 & 5.19 & 76.0 & 71.4 & $0.84 / 99.8$ & severe & $4 Y 3 M / 36.13$ \\
\hline 18 & 60 & $\mathrm{~F}$ & Lung adenocarcinoma; Hypertension; Insomnia & + & + & - & & $24.6 \pm 3.4$ & 5.79 & 2.12 & 48.9 & 31.2 & $0.75 / 83.8$ & No & $1 Y 2 M / 6.51$ \\
\hline 19 & 65 & $\mathrm{~F}$ & Hypertension & + & + & + & & $20.5 \pm 3.3$ & 9.63 & 4.24 & 52.1 & 37.2 & $0.62 / 102.7$ & No & $2 Y 2 M / 12.34$ \\
\hline 20 & 70 & $\mathrm{~F}$ & Hypertension; Major depression & + & + & + & & $25.8 \pm 6.9$ & 6.51 & 3.67 & 64.0 & 54.1 & $0.67 / 92.5$ & mild & $2 Y 9 M / 14.90$ \\
\hline 21 & 62 & $\mathrm{~F}$ & Hypertension & + & + & + & Fatty $^{\mathrm{b}}$ & $23.5 \pm 3.5$ & 5.17 & 3.49 & 53.4 & 31.1 & $0.83 / 74.0$ & mild & $2 Y 10 M / 15.35$ \\
\hline 22 & 61 & $\mathrm{~F}$ & Chronic renal insufficiency; Hyperuricemia & + & Scanty & - & & $23.5 \pm 7.1$ & 4.03 & 2.60 & 59.2 & 49.0 & $2.98 / 17.0$ & No & $3 \mathrm{Y} 1 \mathrm{M} / 18.50$ \\
\hline
\end{tabular}




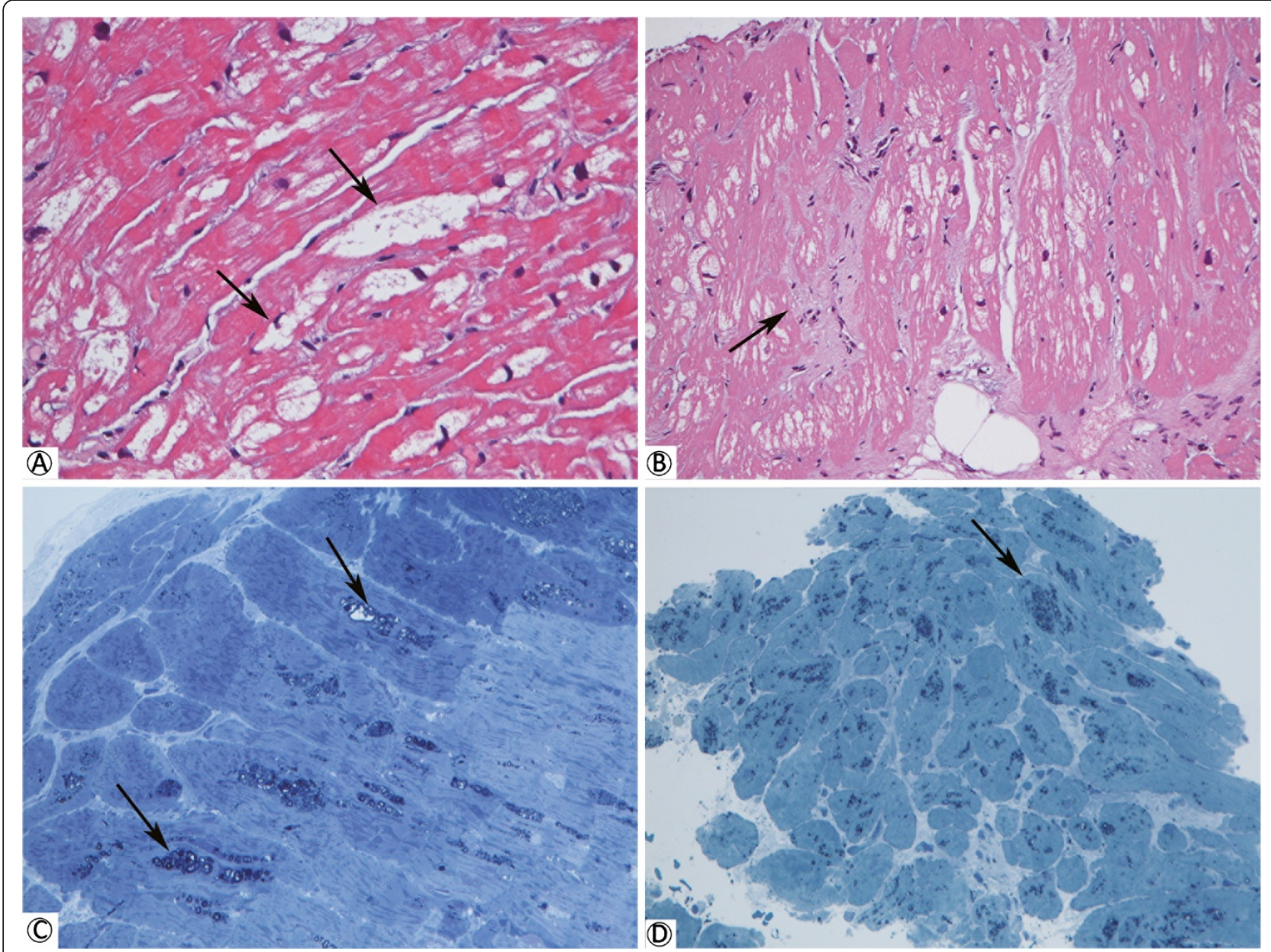

Figure 2 Histologic examination of the cardiomyopathic patients with IVS4 + 919G > A mutation. (A) Endomyocardial biopsy of patient no. 4, who had not received ERT, showed a diffuse vacuolization (arrows) attributed to the lysosomal Gb3 storage and hypertrophic change of cardiomyocytes with hematoxylin and eosin (H\&E) staining. These findings were found in most of the enrolled patients. (B) H\&E staining of the endomyocardium showed mild interstitial fibrosis (arrow) only in one patient (no. 10) and showed similar diffuse vacuolization. Toluidine blue staining of the endomyocardium revealed cytoplasmic granular inclusion (arrow), which is consistent with Gb3 accumulation, in patient no.

4 (C) and patient no. 12 (D) who has received ERT for 2 year and 9 months.

we reviewed the endomyocardial biopsies of large numbers of later-onset Fabry patients with IVS4 + 919G > A mutation to prove this mutation is a real pathogenic mutation.

Cardiac biopsy of classical Fabry disease patients revealed significant $\mathrm{Gb} 3$ accumulation in both endothelium and cardiomyocytes [47-53]. Furthermore, the degree of endothelial Gb3 deposition was even used as a scoring system for monitoring the long-term cardiac therapeutic outcomes of ERT in classic Fabry disease (Thurberg, B.L. et al) [54]. In contrast to classical Fabry disease, cardiac biopsy of the later-onset patients revealed that Gb3 accumulation was confined to the myocardium and did not involve endothelial cells [55]. Other reports have also showed no endothelial Gb3 accumulation in later-onset Fabry disease [56-58]. Therefore, no endothelial Gb3 deposition is expected in later-onset Fabry patients. The cardiac biopsies of our patients showed significant Gb3 accumulation in their cardiomyocytes but not in endothelial cells. It could indicate that IVS4 + 919G > A mutation is a true later-onset Fabry mutation.

Interestingly, a high frequency of hypertension (12 of 22; $54 \%$ ) and hyperlipidemia (7 of 22; $32 \%$ ) were noted among these cardiomyopathic patients with the IVS4 + 919G > A mutation. In this study, we did not check other genetic causes of hypertrophic cardiomyopathy. Actually, the exact causal relationship of these factors with hypertrophic cardiomyopathy in our patients has not established. Because most of our patients did not have significant renal impairment, the hypertension was not likely caused by Fabry renopathy. Several studies have indicated that in addition to renal impairment, hypertension could be caused by the imbalance of the autonomic nervous system in patients with Fabry disease [59]. Further studies are required to clarify the relationship 


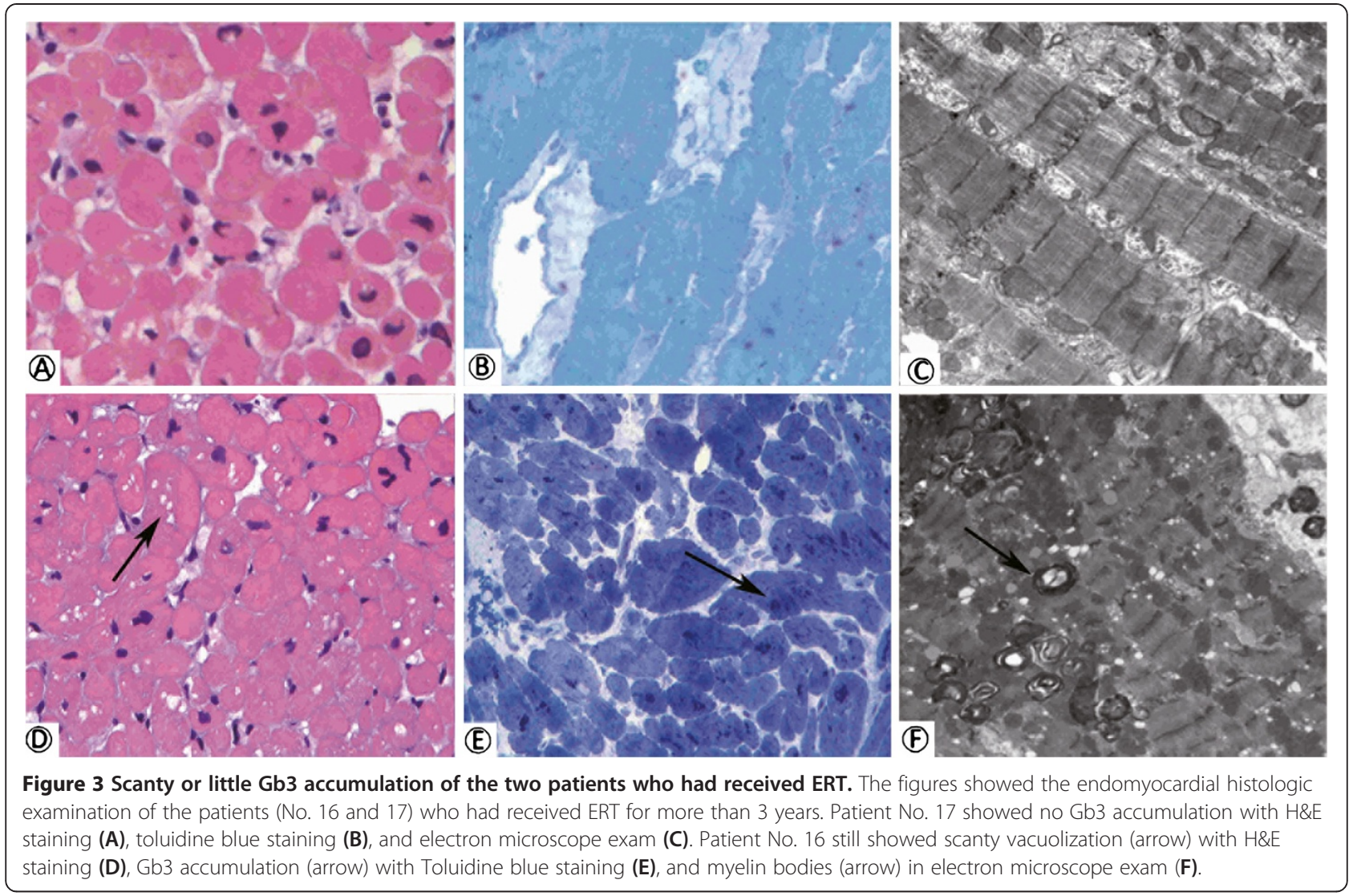

between hypertension and the autonomic nervous imbalance in these patients. Furthermore, hypertension might be a predisposing factor to the development of hypertrophic cardiomyopathy for these later-onset Fabry disease patients, especially for the milder Fabry patients. The finding that four out of our five (80\%) female patients had significant hypertension supports this finding. In addition, several IVS4 + 919G > A patients in our studies were found to be hypertensive. However, due to the small sample size in our study, we cannot make a definite conclusion regarding the relationship between hypertrophic cardiomyopathy and hypertension, more data will need to be collected and analyzed to clarify this point. Regarding predisposing factors for hypertrophic cardiomyopathy in later-onset Fabry patients, a recent important study, reported by Desnick et al. [60], revealed that as many as $20 \%$ of male and $25 \%$ of females with later onset Fabry mutations and hypertrophic cardiomyopathy concurrently have a pathogenic or likely pathogenic mutation of the HCM causing gene. Therefore, in addition to Gb3 accumulation, there should be some predisposing factors contributing to the pathogenesis of later-onset Fabry cardiomyopathy.

Regarding hyperlipidemia in Fabry patients, circulating Gb3 has been proved to be primary transported by LDL and HDL lipoproteins (approximately 60\% and 30\% respectively) [61]. Furthermore, hyperlipidemia was correlated with Fabry disease [62] and selective LDLapheresis was shown to be an effective treatment in a Fabry patient with recurrent strokes [63]. However, the causal relationship between hyperlipidemia and hypertrophic cardiomyopathy of later-onset Fabry patients still needs to be clarified.

Interestingly, only scanty Gb3 accumulation could be found in the cardiomyocytes of our three patients who had received the longest period of ERT. This finding is quite different from several previous studies that showed the histological clearance of Gb3 accumulation from cardiomyocytes is usually poor $[38,49,54,64]$. Does this finding indicate that a longer duration of ERT still has efficacy for Gb3 clearance from cardiomyocytes of lateronset Fabry patients? However, without pre-ERT biopsies, it is impossible to claim ERT was able to clear Gb3 accumulation from the cardiomyocytes of these three patients. More cardiac biopsy data with pre-ERT and post-ERT should be collected to clarify this point.

Recent studies revealed that gadolinium-contrast cardiac MRI is a good method to assess myocardial fibrosis in Fabry disease [34,65-67]. In patients without fibrosis, ERT resulted in a significant reduction in left ventricular mass and improvement of myocardial function. In contrast, patients with mild or severe fibrosis showed a minor 

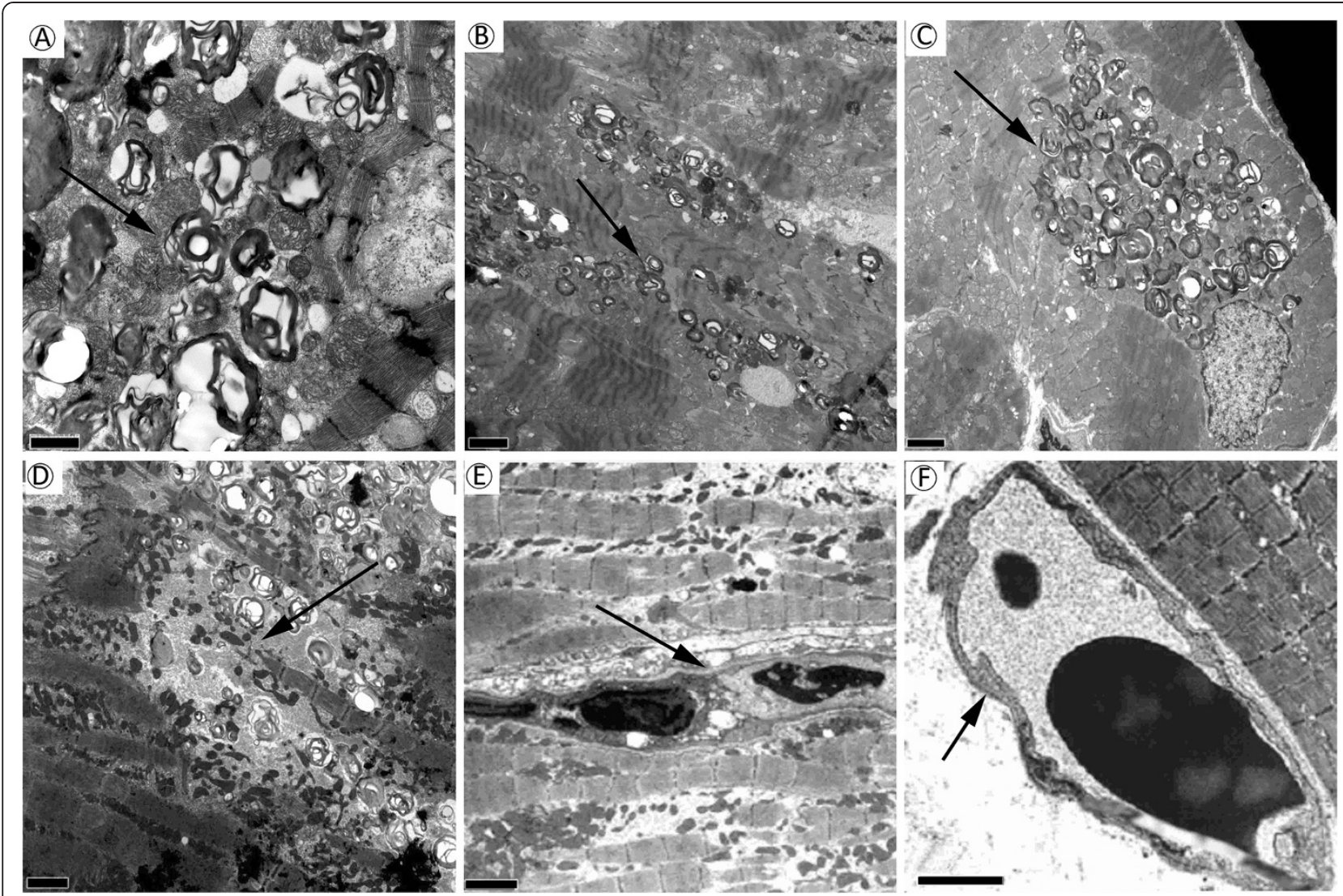

Figure 4 Electron microscopic examination of the patients with left ventricular hypertrophy and IVS4 + 919G > A mutation. Electron microscopic findings of the cardiomyocytes from patient no. 20 showed abundant membrane-bound lamellar myelin bodies ("zebra" or "onionskin" appearance) (arrow) in this figure (A). The myelin bodies (arrow) were also found in most of the patients who received biopsy in this study. Further examples were showed in figure (B) (patient no. 8) and (C) (patient no. 2). Focal loss of myofilament (myofibrillolysis) (arrow), which may be attributed from glycosphinogolipid accumulation and was found in 14 out of 22 case, is presented in figure (D) (patient no. 4). No Gb3 accumulation was found in the capillary endothelium of all these patients. The endothelium (the arrow) were presented in figure (E) (patient No. 2) and (F) (patient No. 3). The scale bars $=2 \mu \mathrm{m}$.

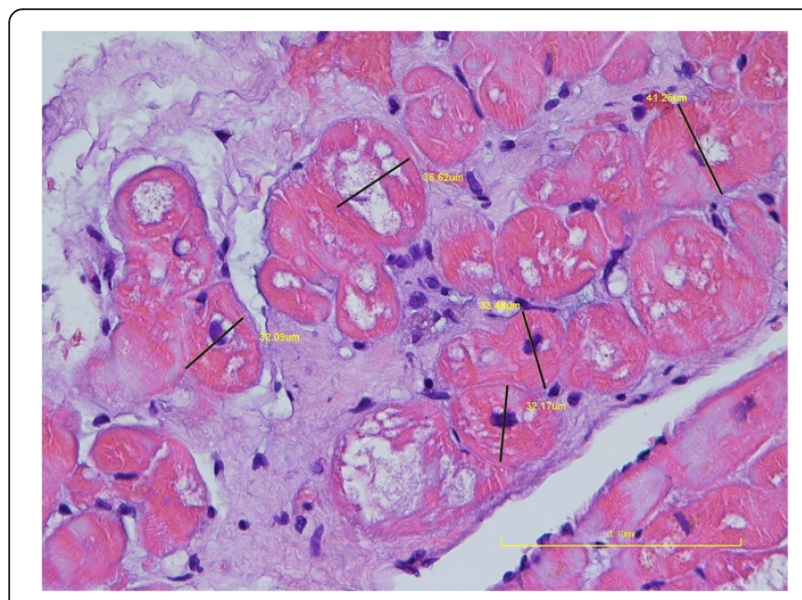

Figure 5 The cardiomyocyte diameter of the patient no. 1 with H\&E staining. Diameters (minor axis) of the cardiomyocyte in transverse sections were obtained by measuring the shortest perpendicular measurement line (dark line), which was placed at the level of nucleus. Five cardiomyocytes were measured and their diameters were also presented in beside the dark line (yellow). reduction in left ventricular hypertrophy and no improvement in myocardial function. In this study, one patient was found to have significant interstitial fibrosis among his cardiomyocytes by H\&E staining of endomyocardial biopsy. However, no significant fibrosis was observed in his heart with late-enhancement technique by gadoliniumcontrast cardiac MRI examination. On the other hand, 6 out of the 20 patients received gadolinium-contrast cardiac MRI examination in this study were found to have severe fibrosis (Table 1), but none of them were found to have any significant cardiac fibrosis in their endomyocardial biopsies. This finding indicates that the histological fibrosis of interventricular septum from right ventricular endomyocardial biopsy does not reflect the severity of the fibrosis of the whole heart; most of the fibrosis that significantly affect heart function is usually located in the left ventricular area in Fabry disease.

In this study, we analyzed the pathologic appearance of cardiomyocytes by endomyocardial biopsies in 22 atypical later-onset Fabry disease patients with the Chinese 
mutation (IVS4 + 919G >A). These data suggest that IVS4 + 919G > A is a real pathogenic mutation with typical Gb3 accumulation and hypertrophic changes in cardiomyocytes. No endothelial Gb3 accumulation was found in cardiac biopsies is consistent with the cardiac pathologic findings of later-onset Fabry disease.

\section{Conclusions}

Fabry disease is a rare lysosomal disorder resulting from a genetic deficiency of $\alpha-$ Gal A. The present study used endomyocardial biopsies to confirm that the Chinese Fabry mutation (IVS4 + 919G > A) is a real pathogenic mutation.

\section{Competing interests}

The authors declare that they have no competing interests.

\section{Authors' contributions}

TRH performed acquisition, statistical analysis, interpretation of data, and drafting of the manuscript. SHS, PCL, and WCY performed the endomyocardial biopsies. FPC and AHY participated in the histopathologic and electron microscopic interpretation. CFY, HCL, and HYL participated in data collection. $\mathrm{CKH}, \mathrm{HJG}, \mathrm{YHH}, \mathrm{HCL}$, and $\mathrm{CCC}$ carried out the biochemistric and genetic analysis. DMN and WCY participated in the design of the study, interpretation of the data and helped to draft the manuscript. All authors contributed in interpreting data, revising drafts of the manuscript and in the approval of the final manuscript.

\section{Acknowledgements}

The authors thank Ms. Tina, Wu and Mr. Hann-Tyng, Chang for their review and suggestion. The authors acknowledge the support from the industryhospital cooperative research project between the Taipei Veterans General Hospital and Shire. Our center has received several research grants regarding Fabry disease from Shire Pharmaceuticals and Excelsior Biopharma. Shire had provided English language editing service for this manuscript. However, Shire had no role in the study design; the collection, analysis and interpretation of data; the writing of the manuscript; or the decision to submit the paper for publication. The opinions are those of the authors.

\section{Funding}

This work was partially supported by the National Science Council, Taiwan (No. NSC-100-2325-B-010-014) and Taipei Veterans General Hospital (No. V101C-129 and V101C-187).

\section{Author details}

${ }^{1}$ Institute of Clinical Medicine, National Yang-Ming University, Taipei, Taiwan. ${ }^{2}$ Department of Pediatrics, Taipei Veterans General Hospital, No. 201, Section 2, Shih-Pai Road, 112 Taipei, Taiwan. ${ }^{3}$ Division of Cardiology, Department of Medicine, Taipei Veterans General Hospital and National Yang-Ming University, School of Medicine, No. 201, Section 2, Shih-Pai Road, 112 Taipei, Taiwan. ${ }^{4}$ Pathology and Laboratory Medicine Department, Taipei Veterans General Hospital, Taipei, Taiwan. ${ }^{5}$ Department of Pediatrics, Mackay Memorial Hospital and Department of Medicine, Mackay Medical College, Taipei, Taiwan. ${ }^{6}$ Taiwan Clinical Trial Consortium in Fabry Disease, Taipei, Taiwan. ${ }^{7}$ Neonatal Screening Center, Chinese Foundation of Health, Taipei, Taiwan. ${ }^{8} \mathrm{College}$ of Medicine, China Medical University, Taichung, Taiwan.

Received: 24 February 2014 Accepted: 19 June 2014 Published: 1 July 2014

\section{References}

1. Meikle PJ, Hopwood JJ, Clague AE, Carey WF: Prevalence of lysosomal storage disorders. JAMA 1999, 281:249-254.

2. Desnick RJ, Wasserstein MP: Fabry disease: clinical features and recent advances in enzyme replacement therapy. Adv Nephrol Necker Hosp 2001, 31:317-339.

3. Desnick RJ, Brady RO: Fabry disease in childhood. J Pediatr 2004, 144:S20-S26.
4. Zarate YA, Hopkin RJ: Fabry's disease. Lancet 2008, 372:1427-1435.

5. Weidemann F, Linhart A, Monserrat L, Strotmann J: Cardiac challenges in patients with Fabry disease. Int J Cardiol 2010, 141:3-10.

6. Bhatia GS, Leahy JF, Connolly DL, Davis RC: Severe left ventricular hypertrophy in Anderson-Fabry disease. Heart 2004, 90:1136.

7. Linhart A: Treatment of Anderson-Fabry disease. Heart 2008, 94:138-139.

8. Kampmann C, Linhart A, Baehner F, Palecek T, Wiethoff CM, Miebach E, Whybra C, Gal A, Bultas J, Beck M: Onset and progression of the Anderson-Fabry disease related cardiomyopathy. Int J Cardio/ 2008, 130:367-373.

9. Imbriaco M, Pisani A, Spinelli L, Cuocolo A, Messalli G, Capuano E, Marmo M, Liuzzi R, Visciano B, Cianciaruso B, Salvatore M: Effects of enzymereplacement therapy in patients with Anderson-Fabry disease: a prospective long-term cardiac magnetic resonance imaging study. Heart 2009, 95:1103-1107.

10. Monserrat L, Gimeno-Blanes JR, Marin F, Hermida-Prieto M, Garcia-Honrubia A, Perez I, Fernandez X, de Nicolas R, de la Morena G, Paya E, Yague J, Egido $\mathrm{J}$ : Prevalence of fabry disease in a cohort of 508 unrelated patients with hypertrophic cardiomyopathy. J Am Coll Cardiol 2007, 50:2399-2403.

11. Nakao S, Takenaka T, Maeda M, Kodama C, Tanaka A, Tahara M, Yoshida A, Kuriyama $\mathrm{M}$, Hayashibe $\mathrm{H}$, Sakuraba $\mathrm{H}$, Tanaka $\mathrm{H}$ : An atypical variant of Fabry's disease in men with left ventricular hypertrophy. $N$ Engl J Med 1995, 333:288-293.

12. Sachdev B, Takenaka T, Teraguchi H, Tei C, Lee P, McKenna WJ, Elliott PM: Prevalence of Anderson-Fabry disease in male patients with late onset hypertrophic cardiomyopathy. Circulation 2002, 105:1407-1411.

13. Kotanko $P$, Kramar R, Devrnja D, Paschke E, Voigtlander T, Auinger $M$, Pagliardini S, Spada M, Demmelbauer K, Lorenz M, Hauser AC, Kofler HJ, Lhotta K, Neyer U, Pronai W, Wallner M, Wieser C, Wiesholzer M, Zodl H, Fodinger M, Sunder-Plassmann G: Results of a nationwide screening for Anderson-Fabry disease among dialysis patients. J Am Soc Nephrol 2004, 15:1323-1329.

14. Tanaka N, Utsumi K, Seta T, Usuda K, Komaba Y, Katsumata T, Katsura KI, Sakamoto S, Katayama Y: Recurrent strokes in a young adult patient with Fabry's disease. Eur J Neurol 2005, 12:486-487.

15. Nance CS, Klein CJ, Banikazemi M, Dikman SH, Phelps RG, McArthur JC, Rodriguez M, Desnick RJ: Later-onset Fabry disease: an adult variant presenting with the cramp-fasciculation syndrome. Arch Neurol 2006, 63:453-457.

16. Nagueh SF: Fabry disease. Heart 2003, 89:819-820.

17. Chong KW, Lu YH, Hsu JH, Lo MY, Hsiao CY, Niu DM: High incidence of cardiac variant of fabry disease in Taiwanese revealed by newborn screening. Taiwan Hum Genet Soc Autumn Symp 2008, 1:92-98.

18. Lin HY, Chong KW, Hsu JH, Yu HC, Shih CC, Huang CH, Lin SJ, Chen CH, Chiang CC, Ho HJ, Lee PC, Kao CH, Cheng KH, Hsueh C, Niu DM: High incidence of the cardiac variant of Fabry disease revealed by newborn screening in the Taiwan Chinese population. Circ Cardiovasc Genet 2009, 2:450-456

19. Hwu WL, Chien YH, Lee NC, Chiang SC, Dobrovolny R, Huang AC, Yeh HY, Chao MC, Lin SJ, Kitagawa T, Desnick RJ, Hsu LW: Newborn screening for Fabry disease in Taiwan reveals a high incidence of the later-onset GLA mutation c.936 + 919G > A (IVS4 + 919G > A). Hum Mutat 2009, 30:1397-1405.

20. Chien YH, Lee NC, Chiang SC, Desnick RJ, Hwu WL: Fabry disease: incidence of the common later-onset a-galactosidase A IVS4+919G $\rightarrow$ A mutation in Taiwanese newborns-superiority of DNA-based to enzyme-based newborn screening for common mutations. Mol Med 2012, 18:780-784.

21. Lin HY, Liu HC, Huang YH, Liao HC, Hsu TR, Shen CI, Li ST, Li CF, Lee LH, Lee PC, Huang CK, Chiang CC, Lin CY, Lin SP, Niu DM: Effects of enzyme replacement therapy for cardiac-type Fabry patients with a Chinese hotspot late-onset Fabry mutation (IVS4 + 919G > A). BMJ Open 2013, 3:e003146. doi:10.1136/bmjopen-2013-003146.

22. Lin HY, Huang $\mathrm{CH}$, Yu HC, Chong KW, Hsu JH, Lee PC, Cheng KH, Chiang CC, Ho HJ, Lin SP, Chen SJ, Lin PK, Niu DM: Enzyme assay and clinical assessment in subjects with a Chinese hotspot late-onset Fabry mutation (IVS4 + 919G-> A). J Inherit Metab Dis 2010, 33:619-624.

23. Buja LM: Evaluation of recombinant alpha-galactosidase $A$ therapy for amelioration of the cardiovascular manifestations of Fabry disease: an important role for endomyocardial biopsy. Circulation 2009, 119:2539-2541.

24. Tai CL, Liu MY, Yu HC, Chiang CC, Chiang H, Suen JH, Kao SM, Huang YH, Wu TJ, Yang CF, Tsai FC, Lin CY, Chang JG, Chen HD, Niu DM: The use of high resolution melting analysis to detect Fabry mutations in 
heterozygous females via dry bloodspots. Clin Chim Acta 2012, 413:422-427.

25. Lee SH, Li CF, Lin HY, Lin CH, Liu HC, Tsai SF, Niu DM: High-throughput detection of common sequence variations of Fabry disease in Taiwan using DNA mass spectrometry. Mol Genet Metab 2014, 111:507-512.

26. Chen CH, Shyu PW, Wu SJ, Sheu SS, Desnick RJ, Hsiao KJ: Identification of a novel point mutation (S65T) in alpha-galactosidase A gene in Chinese patients with Fabry disease: mutations in brief no. 169: online. Hum Mutat 1998, 11:328-330.

27. Rombach SM, Dekker N, Bouwman MG, Linthorst GE, Zwinderman $A H$, Wijburg FA, Kuiper S, Bergh V, Weerman MA, Groener JE, Poorthuis BJ, Hollak CE, Aerts JM: Plasma globotriaosylsphingosine: diagnostic value and relation to clinical manifestations of Fabry disease. Biochim Biophys Acta 1802, 2010:741-748.

28. Aerts JM, Groener JE, Kuiper S, Donker-Koopman WE, Strijland A, Ottenhoff R, van Roomen C, Mirzaian M, Wijburg FA, Linthorst GE, Vedder AC, Rombach SM, Cox-Brinkman J, Somerharju P, Boot RG, Hollak CE, Brady RO, Poorthuis BJ: Elevated globotriaosylsphingosine is a hallmark of Fabry disease. Proc Natl Acad Sci U S A 2008, 105:2812-2817.

29. Liao HC, Huang YH, Chen YJ, Kao SM, Lin HY, Huang CK, Liu HC, Hsu TR, Lin SP, Yang CF, Fann CS, Chiu PC, Hsieh KS, Fu YC, Ke YY, Lin CY, Tsai FJ, Wang CH, Chao MC, Yu WC, Chiang CC, Niu DM: Plasma globotriaosylsphingosine (lysoGb3) could be a biomarker for Fabry disease with a Chinese hotspot late-onset mutation (IVS4 + 919G > A). Clin Chim Acta 2013, 426:114-120.

30. Devereux RB, Alonso DR, Lutas EM, Gottlieb GJ, Campo E, Sachs I, Reichek N: Echocardiographic assessment of left ventricular hypertrophy: comparison to necropsy findings. Am J Cardiol 1986, 57:450-458.

31. Zoccali C, Mallamaci F, Tripepi G, Parlongo S, Cutrupi S, Benedetto FA, Cataliotti A, Malatino LS, Investigators C: Norepinephrine and concentric hypertrophy in patients with end-stage renal disease. Hypertension 2002, 40:41-46.

32. Foppa M, Duncan BB, Rohde LE: Echocardiography-based left ventricular mass estimation. How should we define hypertrophy? Cardiovasc Ultrasound 2005, 3:17.

33. Beer M, Weidemann F, Breunig F, Knoll A, Koeppe S, Machann W, Hahn D, Wanner C, Strotmann J, Sandstede J: Impact of enzyme replacement therapy on cardiac morphology and function and late enhancement in Fabry's cardiomyopathy. Am J Cardiol 2006, 97:1515-1518.

34. Weidemann F, Niemann M, Breunig F, Herrmann S, Beer M, Stork S, Voelker W, Ertl G, Wanner C, Strotmann J: Long-term effects of enzyme replacement therapy on fabry cardiomyopathy: evidence for a better outcome with early treatment. Circulation 2009, 119:524-529.

35. Bates MG, Hollingsworth KG, Newman JH, Jakovljevic DG, Blamire AM, Macgowan GA, Keavney BD, Chinnery PF, Turnbull DM, Taylor RW, Trenell MI, Gorman GS: Concentric hypertrophic remodelling and subendocardial dysfunction in mitochondrial DNA point mutation carriers. Eur Heart $J$ Cardiovasc Imaging 2013, 14:650-658.

36. Cerqueira MD, Weissman NJ, Dilsizian V, Jacobs AK, Kaul S, Laskey WK Pennell DJ, Rumberger JA, Ryan T, Verani MS: Standardized myocardial segmentation and nomenclature for tomographic imaging of the heart: a statement for healthcare professionals from the Cardiac Imaging Committee of the Council on Clinical Cardiology of the American Heart Association. Circulation 2002, 105:539-542.

37. Tracy RE, Sander GE: Histologically measured cardiomyocyte hypertrophy correlates with body height as strongly as with body mass index. Cardiol Res Pract 2011, 2011:658958.

38. Chimenti C, Hamdani N, Boontje NM, DeCobelli F, Esposito A, Bronzwaer JG, Stienen GJ, Russo MA, Paulus WJ, Frustaci A, van der Velden J: Myofilament degradation and dysfunction of human cardiomyocytes in Fabry disease. Am J Pathol 2008, 172:1482-1490.

39. Chimenti C, Padua L, Pazzaglia C, Morgante E, Centurion C, Antuzzi D, Russo MA, Frustaci A: Cardiac and skeletal myopathy in Fabry disease: a clinicopathologic correlative study. Hum Pathol 2012, 43:1444-1452.

40. Tracy RE: Cardiomyocyte size estimated from noninvasive measurements of left ventricular wall thickness and chamber diameter. J Am Soc Hypertens 2012, 6:185-192.

41. Tracy RE: Eccentric may differ from concentric left ventricular hypertrophy because of variations in cardiomyocyte numbers. $J$ Card Fail 2013, 19:517-522.

42. Spada M, Pagliardini S, Yasuda M, Tukel T, Thiagarajan G, Sakuraba H, Ponzone A, Desnick RJ: High incidence of later-onset fabry disease revealed by newborn screening. Am J Hum Genet 2006, 79:31-40.
43. Inoue T, Hattori K, Ihara K, Ishii A, Nakamura K, Hirose S: Newborn screening for Fabry disease in Japan: prevalence and genotypes of Fabry disease in a pilot study. J Hum Genet 2013, 58:548-552.

44. Togawa T, Tsukimura T, Kodama T, Tanaka T, Kawashima I, Saito S, Ohno K, Fukushige T, Kanekura T, Satomura A, Kang DH, Lee BH, Yoo HW, Doi K, Noiri E, Sakuraba H: Fabry disease: biochemical, pathological and structural studies of the alpha-galactosidase A with E66Q amino acid substitution. Mol Genet Metab 2012, 105:615-620.

45. Kitagawa T, Ishige N, Suzuki K, Owada M, Ohashi T, Kobayashi M, Eto Y, Tanaka A, Mills K, Winchester B, Keutzer J: Non-invasive screening method for Fabry disease by measuring globotriaosylceramide in whole urine samples using tandem mass spectrometry. Mol Genet Metab 2005, 85:196-202.

46. Mitobe S, Togawa T, Tsukimura T, Kodama T, Tanaka T, Doi K, Noiri E, Akai Y, Saito Y, Yoshino M, Takenaka T, Saito S, Ohno K, Sakuraba H: Mutant alpha-galactosidase A with M296I does not cause elevation of the plasma globotriaosylsphingosine level. Mol Genet Metab 2012, 107:623-626.

47. Elleder M: Sequelae of storage in Fabry disease-pathology and comparison with other lysosomal storage diseases. Acta Paediatr Suppl 2003, 92:46-53. discussion 45 .

48. Faraggiana T, Churg J, Grishman E, Strauss L, Prado A, Bishop DF, Schuchman E, Desnick RJ: Light- and electron-microscopic histochemistry of Fabry's disease. Am J Pathol 1981, 103:247-262.

49. Hoffmann B: Fabry disease: recent advances in pathology, diagnosis, treatment and monitoring. Orphanet J Rare Dis 2009, 4:21.

50. Tuttolomondo A, Pecoraro R, Simonetta I, Miceli S, Pinto A, Licata G: Anderson-Fabry disease: a multiorgan disease. Curr Pharm Des 2013, 19:5974-5996.

51. Germain DP: Fabry disease. Orphanet J Rare Dis 2010, 5:30.

52. Fischer EG, Moore MJ, Lager DJ: Fabry disease: a morphologic study of 11 cases. Mod Pathol 2006, 19:1295-1301.

53. Eng CM, Guffon N, Wilcox WR, Germain DP, Lee P, Waldek S, Caplan L, Linthorst GE, Desnick RJ: Safety and efficacy of recombinant human alpha-galactosidase A-replacement therapy in Fabry's disease. N Engl J Med 2001, 345:9-16.

54. Thurberg BL, Fallon JT, Mitchell R, Aretz T, Gordon RE, O'Callaghan MW: Cardiac microvascular pathology in Fabry disease: evaluation of endomyocardial biopsies before and after enzyme replacement therapy. Circulation 2009, 119:2561-2567.

55. von Scheidt W, Eng CM, Fitzmaurice TF, Erdmann E, Hubner G, Olsen EG, Christomanou H, Kandolf R, Bishop DF, Desnick RJ: An atypical variant of Fabry's disease with manifestations confined to the myocardium. $N$ Engl J Med 1991, 324:395-399.

56. Frustaci A, Chimenti C, Ricci R, Natale L, Russo MA, Pieroni M, Eng CM, Desnick RJ: Improvement in cardiac function in the cardiac variant of Fabry's disease with galactose-infusion therapy. N Engl J Med 2001, 345:25-32.

57. Takenaka T, Teraguchi H, Yoshida A, Taguchi S, Ninomiya K, Umekita Y, Yoshida H, Horinouchi M, Tabata K, Yonezawa S, Highchi K, Nakao S, Anan $R$, Minagoe $S$, Tei $C$ : Terminal stage cardiac findings in patients with cardiac Fabry disease: an electrocardiographic, echocardiographic, and autopsy study. J Cardiol 2008, 51:50-59.

58. Germain DP, Shabbeer J, Cotigny S, Desnick RJ: Fabry disease: twenty novel alpha-galactosidase A mutations and genotype-phenotype correlations in classical and variant phenotypes. Mol Med 2002, 8:306-312.

59. Eric $A$, Aurore $S, R$ F, Vincent $P$, Jean-Claude $B$ : Autonomic nervous system activity in patients with Fabry disease. Open J Intern Med 2012, 2:116-122.

60. Desnick RJ, Doheny D: Targeted sequencing of over 4000 hypertrophic cardiomyopathy (HCM) patients for mutations causing HCM and Fabry disease: $\mathrm{HCM}$ mutations frequent n patients with GLA later0onset mutations, polymorphisms, and variant. Mol Genet Adn Metab 2014, 111:s36-s37.

61. Desnick RJ, loannou YA, Eng CM: a-Galactosidase a deficiency: Fabry disease (2001). Metab Mol Bases Inherited Dis 2001, 8th edn:3373-3774.

62. Cartwright DJ, Cole AL, Cousins AJ, Lee PJ: Raised HDL cholesterol in Fabry disease: response to enzyme replacement therapy. I Inherit Metab Dis 2004, 27:791-793.

63. Utsumi K, Seta T, Katsumata T, Komaba Y, Igarashi H, Katsura Kl, lino Y, Katayama Y: Effect of selective LDL-apheresis in a Fabry patient with recurrent strokes. Eur J Neurol 2006, 13:429-430. 
64. Tondel C, Bostad L, Larsen KK, Hirth A, Vikse BE, Houge G, Svarstad E: Agalsidase benefits renal histology in young patients with Fabry disease. J Am Soc Nephrol 2013, 24:137-148.

65. Weidemann F, Breunig F, Beer M, Sandstede J, Stork S, Voelker W, Ertl G, Knoll A, Wanner C, Strotmann JM: The variation of morphological and functional cardiac manifestation in Fabry disease: potential implications for the time course of the disease. Eur Heart J 2005, 26:1221-1227.

66. Sado DM, White SK, Piechnik SK, Banypersad SM, Treibel T, Captur G, Fontana M, Maestrini V, Flett AS, Robson MD, Lachmann RH, Murphy E, Mehta A, Hughes D, Neubauer S, Elliott PM, Moon JC: Identification and assessment of Anderson-Fabry disease by cardiovascular magnetic resonance noncontrast myocardial T1 mapping. Circ Cardiovasc Imaging 2013, 6:392-398.

67. Koeppe S, Neubauer H, Breunig F, Weidemann F, Wanner C, Sandstede J, Machann W, Hahn D, Kostler H, Beer M: MR-based analysis of regional cardiac function in relation to cellular integrity in Fabry disease. Int $\mathrm{J}$ Cardiol 2012, 160:53-58.

doi:10.1186/1750-1172-9-96

Cite this article as: Hsu et al: Endomyocardial biopsies in patients with left ventricular hypertrophy and a common Chinese later-onset fabry mutation (IVS4 + 919G > A). Orphanet Journal of Rare Diseases 2014 9:96.

\section{Submit your next manuscript to BioMed Central and take full advantage of:}

- Convenient online submission

- Thorough peer review

- No space constraints or color figure charges

- Immediate publication on acceptance

- Inclusion in PubMed, CAS, Scopus and Google Scholar

- Research which is freely available for redistribution

Submit your manuscript at www.biomedcentral.com/submit 